\title{
Familias Multiproblemáticas y en Riesgo Social: Características e Intervención
}

\section{Multiproblem Families at Social Risk: Characteristics and Intervention}

\author{
Esteban Gómez \\ Pontificia Universidad Católica de Chile \\ Sociedad Protectora de la Infancia \\ María Magdalena Muñoz \& Ana María Haz \\ Pontificia Universidad Católica de Chile
}

\begin{abstract}
El artículo comprende la familia multiproblemática como un sistema vulnerable de alto riesgo, tanto por la acción de sus mecanismos internos, como de fuerzas sociales que potencian su disfuncionalidad. Tras una extensa revisión bibliográfica, los autores proponen como sus características centrales la polisintomatología y crisis recurrentes, la desorganización, el abandono de las funciones parentales y el aislamiento, focalizando específicamente en aquellas familias en situación de riesgo social. En una segunda parte, se desarrolla un esquema de intervención profesional, que incorpora la función de los servicios sociales y su relación potencialmente nociva con estas familias, el trasfondo epistemológico y los contextos en que se despliega la intervención, y las principales estrategias sugeridas para implementar buenas prácticas en el trabajo profesional.
\end{abstract}

Palabras Clave: familia multiproblemática, riesgo social, pobreza, servicios sociales, intervención psicosocial.

\begin{abstract}
The paper understands multiproblem family as a vulnerable system of high risk, in part due to their internal mechanisms and in part by the action of social forces that reinforce their malfunction. After an extensive bibliographic review, the authors propose as its central characteristics, poly-symptomatology and recurrent crisis, disorganization, abandonment of parental functions and isolation, focusing the discussion specifically on those families at social risk. In a second part, the article develops a schema of professional intervention, that includes the function of social services and their potentially noxious relationship with these families, the epistemological background and the contexts on which the intervention unfolds, and the main strategies suggested to implement good practices in the professional work.
\end{abstract}

Keywords: multiproblem family, social risk, poverty, social services, psychosocial intervention.

Ligado a las transformaciones socioculturales, políticas y económicas acaecidas en el último siglo, se ha debilitado el pacto tradicional que vinculaba

Esteban Gómez, programa de Magíster en Psicología Clínica, Pontificia Universidad Católica de Chile; Sociedad Protectora de la Infanciai.

Magdalena Muñoz, programa de Magíster en Psicología Clínica, Pontificia Universidad Católica de Chile.

Ana María Haz, Escuela de Psicología CEES UC, Pontificia Universidad Católica de Chile.

La correspondencia de este artículo debe dirigirse a Esteban Gómez: eagomez@puc.cl o egomez@protectora.cl

La Sociedad Protectora de la Infancia es una organización privada sin fines de lucro con 113 años de historia, cuya misión es contribuir al mejoramiento de las condiciones de vida de la infancia en Chile, en particular de aquellos niños y jóvenes en situación de vulnerabilidad y escasos recursos, a través de programas educacionales y sociales. Recientemente ha desarrollado un Marco Conceptual Orientador (para guiar el diseño, intervención y evaluación de resultados) que incluye cinco componentes: apego, resiliencia, organización familiar, red y necesidades básicas, sirviendo de inspiración para esta propuesta. gobierno, sociedad y familia en un proyecto común (Haz \& Matus, 2006). Esto ha generado en muchas comunidades un ambiente de incertidumbre, desconcierto y pérdida de los referentes tradicionales sobre «cómo se debe vivir la vida» (Bauman, 2001), lo que aunado a dinámicas de exclusión social cada vez más arraigadas, ha impactado a numerosas familias vulnerables, destacando por su extrema complejidad la Familia Multiproblemática (Linares, 1997).

Estas familias no cuentan con herramientas suficientes para afrontar las demandas de éxito ligadas a una cultura hedonista y de consumo (Ditzel \& Maldonado, 2004), que exige asumir la responsabilidad por las propias historias de vida (Ehrenberg, 1999), y que mide la valía personal según criterios difícilmente alcanzables. Así, al encontrarse mayoritariamente en contextos caracterizados por factores de riesgo psicosocial y deprivación sociocultural crónica, se refuerza el ciclo de marginación, disfuncionalidad, crisis y desesperanza que es común observar en ellas. 
Durante las décadas pasadas se han implementado numerosas iniciativas para ayudar a estas familias, implicando inicialmente a voluntarios y organizaciones no profesionales, y más recientemente, a la comunidad profesional y académica. En su mayoría, estos esfuerzos no han tenido un impacto duradero, pero han arrojado valiosa información desde la cual es imperativo seguir construyendo conocimiento y propuestas de intervención.

El presente artículo busca aportar en esta línea, y se ha estructurado desde una amplia revisión bibliográfica en diversas bases de datos (ProQuest, PsycINFO, OCLC-FirstSearch y SciELO, entre otras), seleccionando las referencias según aportaran información sobre las características de la familia multiproblemática y/o la intervención con éstas.

\section{Características}

Dentro de los aspectos más difíciles del trabajo con familias multiproblemáticas (en adelante, FMP), está el comprender su funcionamiento y anticipar los escenarios posibles de intervención. Estas familias sorprenden a los equipos con modulaciones sistémicas que no encajan en los modelos y metodologías tradicionales. Por ello, es indispensable desarrollar marcos orientadores específicos, que permitan al profesional distinguir rasgos comunes en la variedad, proyectando su abordaje técnico en conocimiento del impacto de cada una de estas variables.

Como esquema organizador de la literatura respecto a las FMP, los autores del presente artículo proponen cuatro ejes descriptivos, que si bien se manifiestan entrelazados en la práctica, son útiles de distinguir conceptualmente: (1) Polisintomatología y crisis recurrentes; (2) Desorganización; (3) Abandono de las Funciones Parentales; y (4) Aislamiento.

\section{Polisintomatología y Crisis Recurrentes}

Estas familias no presentan un síntoma particular, sino que una cadena de problemas y factores de estrés (Matos \& Sousa, 2004), entre los que pueden mencionarse negligencia, alcoholismo, violencia intrafamiliar, abuso de sustancias y depresión. Sharlin y Shamai (1995) señalan que este patrón es de tal importancia, que las FMP debiesen reconceptualizarse como "familias en extremo distrés" ofamilias multi-estresadas.

En esta línea, Walsh (2004) ha definido tres características centrales en las FMP, todas asociadas al eje de polisintomatología: (a) presencia de problemas múltiples, de gran complejidad y gravedad; (b) más de un portador de sintomatología; y (c) episodios recurrentes de crisis individuales y familiares. Según Casas (1998 citado en Navarro, 2002), estas crisis recurrentes, que marcan la dinámica de las FMP, pueden aparecer como reacción a:

(a) Dificultades de carácter material o físico, como cesantía, enfermedades crónicas, etc.;

(b) Relaciones conflictuadas con el entorno circundante, expresadas como marginación social o demandas de adaptación a contextos violentos o estresantes, y;

(c) Relaciones conflictuadas al interior del sistema familiar, y que adoptan la forma de violencia intrafamiliar, abuso sexual, negligencia, etc.

Cuando estas condiciones no reciben un apoyo e intervención adecuados, se cronifica y refuerza de forma poderosa la polisintomatología característica de estas familias, en un ciclo deteriorante de condiciones adversas-falta de apoyo y de recursos-crisis- y sintomas múltiples. Ahora bien, no basta solamente con dar apoyo, sino que éste debe calzar con la necesidad particular que ha desencadenado la crisis para ser efectivo (Juby \& Rycraft, 2004).

\section{Desorganización}

El segundo eje pone el énfasis en la desorganización que define la dinámica de estas familias, particularmente en torno a dos aspectos: (a) una estructura caótica; y (b) una comunicación disfuncional. Conceptos que apuntan en esta línea son los de familia "suborganizada" de Aponte (1976, 1981 citado en Cancrini, De Gregorio \& Nocerino, 1997) o familia "desorganizada" de Minuchin et al. (1967a; 1967b citado en Cancrini et al., 1997).

Vega (1997) ha mencionado como características de la estructura de estas familias el ser mayoritariamente monoparentales y presentar una notoria dispersión de sus miembros. Asimismo, dicha estructura se caracteriza por numerosas rupturas y reconstituciones que configuran genogramas complejos y a veces confusos (Linares, 1997; Matos \& Sousa, 2004; Sousa \& Eusébio, 2005).

En estas familias existen conflictos en el desarrollo de los roles y la delimitación de los subsistemas (Cancrini et al., 1997). Presentan una elevada porosidad de sus fronteras, con límites generalmente difusos y problemas de diferenciación, lo que facilita la transmisión transgeneracional de los patrones de 
desorganización y multi-problematicidad, como lo muestra el estudio de Hurst, Sawatsky y Pare (1996).

Se ha detectado la presencia de una jerarquía caótica y una reducción de las reglas y normas al mínimo (Sousa \& Eusébio, 2005), sin rutinas claras ni criterios consensuados sobre qué está permitido y qué no en la familia. El poder no se concentra "en un miembro determinado de la familia y como consecuencia de ello la interacción familiar tendrá carácter caótico, estableciéndose el control a través de medios indirectos y encubiertos" (Navarro, 2002, p. 26); o a través de la descarga en el acto agresivo (Sousa \& Eusébio, 2005). Además, los hijos pueden implementar una "prueba de límites" en escalada, que busca definir de alguna forma un bosquejo de estructura familiar, al costo de invertir muchas veces los roles familiares.

El segundo aspecto de la desorganización familiar dice relación con la comunicación, que resulta caótica y empobrecida en su intercambio de información (Malagoli Togliatti, 1985 citado en Cancrini et al., 1997). Se observan patrones incongruentes de comunicación verbal y no verbal, lo que se manifiesta como ambivalencia relacional, habitualmente anclada en una historia de desórdenes vinculares transgeneracionales (Hurst et al., 1996; Sousa \& Eusébio, 2005).

La ambivalencia e incongruencia encuadran una dificultad para "leer" adecuadamente los matices e implicancias de un mensaje y para desarrollar empatía en la comunicación. En situaciones de resolución de conflictos, tienden a interpretarse las "señales" comunicativas como muestras de rechazo o abandono. Como el lenguaje ha perdido su capacidad mediadora se instalan los golpes y gritos, o la autoagresión y autodestrucción, el acting out como solución de "punto final" y de descarga de la tensión acumulada.

\section{Abandono de las Funciones Parentales}

La investigación en FMP ha revelado que éstas tienden a abandonar sus funciones parentales (Cancrini et al., 1997; Navarro, 2002), mostrando en muchos casos un grado elevado de incompetencia parental y negligencia (Barudy, 1998), que llevado al extremo (por ejemplo, ligado a dinámicas de maltrato infantil grave) puede acarrear la inhabilitación legal de los padres y la salida del niño de su familia.
Se han distinguido tres funciones parentales básicas (Barudy \& Dantagnan, 2005): la nutriente, la socializadora y la educativa. El debilitamiento de las finalidades socializadoras y educativas en la FMP altera en los niños y niñas su capacidad de inserción y adaptación social, al no facilitar la internalización de "normas y valores culturales, lo cual inhibe el desarrollo de la consideración y el respeto a la sociedad por parte del niño y sitúa a éste en posición de conflicto con su entorno" (Linares, 1997, p. 32).

El debilitamiento de la función nutriente en la FMP, principalmente de tipo emocional, obstaculiza el desarrollo de un apego seguro (Cyrulnik, 2002) y lleva a un riesgo significativamente aumentado de padecer diversos trastornos biopsicosociales (Glaser, 2002). Numerosos estudios han mostrado en niños físicamente maltratados la predominancia de apego evitativo, mientras que niños que han sufrido negligencia física muestran predominancia de un apego ansioso/ambivalente (Finzi, Ram, Har-Even, Shnit \& Weizman, 2001); finalmente, los niños y niñas que han recibido una combinación de diversas formas de maltrato, abuso $\mathrm{y}$ negligencia muestran predominantemente un estilo de apego desorganizado o ansioso/evitativo (Hughes, 2004). Asimismo, la investigación en niños que han sufrido negligencia y/o maltrato emocional, ha mostrado la presencia de diversas dificultades posteriores en la infancia, adolescencia y vida adulta, observándose trastornos emocionales (como baja autoestima, ansiedad o estrés), desórdenes conductuales (como irresponsabilidad, oposicionismo y comportamientos antisociales), bajo desempeño y ausentismo escolar, agresividad y/o aislamiento social, en la mayoría de estos niños (Glaser, 2002).

Este abandono de las funciones parentales aparece vinculado en muchos casos a dinámicas de entrega y/o abandono de los hijos (Vega, 1997), por cuanto el subsistema parental delega sistemáticamente el cuidado de sus hijos en terceros (vecinos, familiares, profesionales).

La intervención sobre las condiciones de vulneración que demandan la salida del niño de su hogar, se dificulta muchísimo cuando se traspasa la línea del abandono de las funciones parentales, con bajas tasas de éxito (25\%-70\%) en la mayoría de los diversos programas que intervienen después de este punto (de Paúl, Múgica \& Alday, 1997). Es por ello que comprender esta variable en la intervención, resulta crucial si los profesionales 
hemos de ayudar a estas familias a permanecer unidas sin vulnerar el desarrollo sano de sus niños y niñas.

\section{Aislamiento}

Por último, el cuarto eje tiene que ver con el aislamiento. Para aludir a esta característica, se les ha denominado "familias aisladas" (Powell \& Monahan, 1969 citado en Cancrini et al., 1997), o "familias excluidas" (Thierny, 1976 citado en Cancrini et al., 1997), destacando su distanciamiento, físico y emocional, de la familia extensa y la red de apoyo social e institucional, encontrándose así carentes de soporte frente a las crisis que atraviesan. Cabe destacar que la variable "aislamiento social" o "falta de apoyo social" ha sido identificada en numerosas investigaciones como un factor gravitante en la etiología del maltrato infantil (Gracia \& Musitu, 1993; Moncher, 1995). Asimismo, la red de apoyo ha sido asociada a numerosos factores protectores de crucial importancia (Sluzki, 1996); de ahí que su precariedad y escasa disponibilidad en las FMP influya en la gravedad y multiplicidad de los síntomas.

Incluso cuando la familia tiene una red social disponible, existe una tendencia a la inestabilidad y fragilidad en estos vínculos, al desarrollarse sobre un trasfondo de carencias en las habilidades sociales necesarias para mantener sus relaciones interpersonales en el tiempo (Juby \& Rycraft, 2004; Sousa, 2005). Asimismo, se ha visto que el apoyo de la familia extensa, cuando está presente, se caracteriza por anular sus efectos potencialmente positivos por acompañarse de críticas y descalificaciones frecuentes (Moncher, 1995).

Estas redes suelen ser precarias y constituidas por parientes y conocidos que no proveen el tipo de ayuda que requieren los padres en su parentalidad (Moncher, 1995). En contraposición a una dinámica resiliente, los miembros de esta red generalmente comparten y refuerzan las normas y conductas de parentalidad negligente (Juby \& Rycraft, 2004; Sousa, 2005). Esto explicaría en parte hallazgos recientes que controvierten el análisis de que la negligencia parental ocurre principalmente en condiciones de aislamiento social; la negligencia puede ocurrir tanto en condiciones de aislamiento, como en presencia de redes informales que contemplan indiferentemente o hasta estimulan en esa dirección (Roditti, 2005).

\section{Familias Multiproblemáticas y Pobreza}

De acuerdo a un estudio de Aldaz-Carroll y Moran (2001), sobre la transmisión transgeneracional de la pobreza en 16 países de Latinoamérica, los "factores familiares" (como baja escolaridad, estructura familiar disfuncional o presencia de violencia doméstica) son centrales en la comprensión y abordaje de la pobreza. Así, fortalecer la familia es una condición sine qua non de superación de la pobreza.

Las FMP no son exclusivas de un entorno de pobreza y deprivación, ya que pueden encontrarse en cualquier contexto social, cultural y económico (Matos \& Sousa, 2004; Sousa \& Eusébio, 2005). Sin embargo, si a la calidad de "multiproblemática" se le suma el de "pobreza dura" (Rozas, 1999), los efectos perjudiciales para sus miembros se tornan estructurales y de difícil modificación.

La familia en pobreza dura se encuentra atrapada en una especie de laberinto social, donde a cada movimiento pareciera hundirse más y más. Los distintos actores que van conformando la familia, padre, madre, hijos, abuelos, amigos, presentan inhabilidades sociales que en distintos momentos les impiden salvar obstáculos fundamentales para su integración social (...) impactando a los niños. Estos últimos, sin herramientas intelectuales, culturales, sociales, ven fragmentado su proyecto vital, reconstituyendo el ciclo de la pobreza. (Rozas, 1999, p. 86)

La pobreza emerge como una variable de particular influencia en la cronificación de conductas parentales negligentes (si bien no debe igualarse pobreza a negligencia, ni a las otras características señaladas previamente). Los efectos indirectos de la pobreza que potencian el desarrollo de dinámicas negligentes y/o maltratadoras, incluyen historias de traumatismo y deprivación, condiciones de adversidad crónicas, mayor cantidad de variables de estrés parental, aislamiento social, monoparentalidad, embarazos adolescentes, y vivir en comunidades que son peligrosas, violentas y que carecen del capital social necesario para apoyar la crianza de los hijos (Woodward \& Fergusson, 2002).

Producto de esta dinámica, que entrelaza un funcionamiento inadecuado, carencia de recursos y condiciones de vida adversas, las FMP en riesgo social terminan por involucrarse casi ineludiblemente con agentes de control externos. Con esto, terminarían por disminuirse aun más sus competencias y habilidades de enfrentamiento (Matos \& Sousa, 2004; 
Minuchin, Colapinto \& Minuchin, 2000), delegando sus cuidados parentales en diversos servicios de la comunidad (Vega, 1997).

\section{Intervención}

\section{Familias Multiproblemáticas y Servicios Sociales}

La mayor parte de los programas sociales trabaja con familias multiproblemáticas en situación de pobreza y riesgo social (Juby \& Rycraft, 2004; Walsh, 2004). A pesar de los esfuerzos realizados durante décadas, y de algunas experiencias aisladas de intervención exitosa, la mayoría de estas iniciativas no ha decantado hacia resultados positivos duraderos con estas familias.

Las razones de esta dificultad son diversas; Walsh critica que estos servicios tienden a "basarse en las deficiencias, concentrarse en el individuo y ser fragmentarios, reactivos a las crisis, inaccesibles y definidos por los profesionales para los clientes" (Walsh, 2004, p. 329).

Colapinto (1995), por otro lado, ha puesto de relieve la disolución de los procesos familiares, entendida como la transferencia de las funciones parentales y familiares hacia los servicios sociales, y que ocurre producto de la multi-asistencia sostenida en el tiempo y la invasión progresiva en todas las áreas de la vida familiar por parte de los profesionales. Estas intervenciones "son a veces necesarias, pero siempre desarticulan las estructuras familiares. Se llevan a cabo sin tomar en cuenta los vínculos emocionales positivos, y anulan recursos potenciales" (Minuchin et al., 2000, p. 36).

Además, se ha mostrado que mientras estas familias presentan una amplia gama de problemas complejos y graves, las agencias sociales se encuentran organizadas casi invariablemente por áreas de intervención (Matos \& Sousa, 2004; Sousa, 2005). En consecuencia, la compleja matriz de conflictos familiares involucra y afecta diferentes sistemas simultáneamente: al sistema escolar en situaciones de ausentismo, desórdenes conductuales y problemas de integración escolar; al sistema de salud en casos de adicción a drogas y alcohol, psicosis, depresiones, etc.; al sistema judicial en situaciones de violencia intrafamiliar, abandono, vulneración de derechos, y así sucesivamente.

De esta forma, en corto tiempo acumulan interacciones con gran cantidad de profesionales e instituciones, reforzando su dependencia y desarticulando la eficiencia de las intervenciones (Matos \& Sousa,
2004; Minuchin et al., 2000). Además, es frecuente que estas familias tornen difícil la colaboración coordinada de los profesionales de un mismo equipo y aun más infructuoso el trabajo sincrónico de equipos de diferentes servicios (Vega, 1997).

Finalmente, se ha remarcado que la petición de ayuda rara vez proviene de la propia familia, siendo usualmente otro sistema de la red (policía, escuela, vecinos) quien da la alerta y/o deriva a diversas organizaciones de ayuda (Matos \& Sousa, 2004), lo que implica que la motivación al cambio rara vez se encuentra garantizada al inicio de la intervención profesional.

\section{Trasfondo Epistemológico de la Intervención}

Siguiendo la propuesta de Vega (1997) respecto al trasfondo epistemológico óptimo para enmarcar la intervención con FMP, proponemos una síntesis en torno a tres enfoques interrelacionados: (1) una perspectiva constructivista de la realidad humana; (2) una visión ecosistémica de los procesos familiares; y (3) una comprensión del cambio como proceso discontinuo.

En primer lugar, la intervención con FMP se ve fortalecida cuando se desarrolla desde una mirada constructivista de la realidad humana, que no es igual a decir "todo vale", pero que sí se distingue de un enfoque objetivista, al aceptar como válidas las construcciones sociales propias de la comunidad en que se desenvuelven las familias. Dichos constructos son el marco en el cual las pautas de la familia cobran un sentido específico; si el profesional es capaz de desarrollar una actitud de interés y respeto por este marco, gana en apertura y empatía, facilitándose el manejo de las disonancias ineludibles al trasfondo sociocultural del profesional. La noción de la realidad humana como construcción social tiene como consecuencia que el profesional debe "flexibilizar sus propias nociones de familia y hogar para poder intervenir" (Vega, 1997, p. 169).

Sin embargo, esto no implica eliminar las consideraciones sobre el respeto por una ética de mínimos, "sobre cuestiones de justicia, exigibles moralmente a todos los ciudadanos (...) por debajo de las cuales no puede caer una sociedad sin caer a la vez "bajo mínimos" de moralidad" (Cortina, 1998, p. 117).

En segundo lugar, una visión global multidimensional o ecosistémica, sustentada en los conceptos de causalidad circular, complejidad y emergencia de fenómenos con cualidad de novedosos permite, al trabajar con sistemas humanos, buscar conexiones y relaciones más allá de las comprensiones reduc- 
Tabla 1

Contextos de Intervención

Contextos de Intervención

\begin{tabular}{|c|c|c|c|c|c|c|}
\hline & Asistencial & Consulta & Terapéutico & Evaluación & Control & Informativo \\
\hline Palabra Clave & Ayuda & Consejo & $\begin{array}{l}\text { Co-creación } \\
\text { de alternativas }\end{array}$ & Diagnóstico & Regulación & Enseñanza \\
\hline Objetivo & $\begin{array}{l}\text { Justicia Social } \\
\text { Satisfacción } \\
\text { de necesidades } \\
\text { básicas }\end{array}$ & $\begin{array}{l}\text { Toma de } \\
\text { decisiones } \\
\text { Clarificación }\end{array}$ & $\begin{array}{l}\text { Cambio de } \\
\text { perspectiva } \\
\text { Vivir de otra } \\
\text { forma }\end{array}$ & $\begin{array}{l}\text { Habilitación } \\
\text { Inhabilitación } \\
\text { Delimitar áreas } \\
\text { de intervención }\end{array}$ & $\begin{array}{l}\text { Cumplimiento } \\
\text { de leyes } \\
\text { Protección de } \\
\text { derechos }\end{array}$ & Aprender \\
\hline Metodología & $\begin{array}{l}\text { Asistencialismo } \\
\text { Intervención } \\
\text { de red }\end{array}$ & Consejería & Psicoterapia & $\begin{array}{l}\text { Psicodiagnóstico } \\
\text { Peritaje forense } \\
\text { Evaluación } \\
\text { social }\end{array}$ & $\begin{array}{l}\text { Intervención } \\
\text { coactiva }\end{array}$ & $\begin{array}{l}\text { Psicoeducación } \\
\text { Socioeducación }\end{array}$ \\
\hline Modalidad & $\begin{array}{l}\text { Entrevistas } \\
\text { Visitas } \\
\text { domiciliarias } \\
\text { Coordinaciones } \\
\text { de Red }\end{array}$ & $\begin{array}{l}\text { Ciclo acotado } \\
\text { de entrevistas } \\
\text { con foco } \\
\text { temático con } \\
\text { un individuo o } \\
\text { grupo }\end{array}$ & $\begin{array}{l}\text { Conjunto de } \\
\text { sesiones de } \\
\text { terapia con } \\
\text { individuos, } \\
\text { familias, } \\
\text { parejas o } \\
\text { grupos }\end{array}$ & $\begin{array}{l}\text { Entrevistas } \\
\text { Aplicación de } \\
\text { pruebas } \\
\text { Documentación }\end{array}$ & $\begin{array}{l}\text { Entrevistas } \\
\text { Visitas } \\
\text { domiciliarias } \\
\text { Citación a } \\
\text { tribunales }\end{array}$ & $\begin{array}{l}\text { Talleres } \\
\text { Charlas }\end{array}$ \\
\hline $\begin{array}{l}\text { Técnicas } \\
\text { típicas }\end{array}$ & $\begin{array}{l}\text { Gestión de } \\
\text { recursos } \\
\text { Entrega de } \\
\text { beneficios }\end{array}$ & $\begin{array}{l}\text { Focalización } \\
\text { Co-reflexión } \\
\text { Propuestas de } \\
\text { acción }\end{array}$ & $\begin{array}{l}\text { Vinculación } \\
\text { Narraciones } \\
\text { alternativas } \\
\text { Prescripciones }\end{array}$ & $\begin{array}{l}\text { Identificar } \\
\text { recursos y } \\
\text { falencias }\end{array}$ & $\begin{array}{l}\text { Seguimiento } \\
\text { Coacción }\end{array}$ & $\begin{array}{l}\text { Exposición de } \\
\text { información } \\
\text { novedosa } \\
\text { Compartir } \\
\text { experiencias } \\
\text { Modelar }\end{array}$ \\
\hline
\end{tabular}

Nota. Tabla adaptada de Lamas (1997).

cionistas de un problema. Esta visión ecosistémica incluye vislumbrar la necesaria interdependencia entre los diversos actores implicados en el proceso de ayuda y el lugar que ocupa el profesional o equipo, para desbloquear cadenas inoperantes de intervenciones incongruentes entre sí y de carácter crónico (Vega, 1997).

En tercer lugar, una concepción del cambio como un proceso discontinuo (contrapuesto a una noción de cambio lineal, gradual, progresivo y acumulativo) es fundamental, ya que, al trabajar con familias multiproblemáticas,

...el profesional necesita tener una guía clara de las posibilidades de cambio en estas familias. Sin ello, no es posible desencadenar, conducir y usar el cambio. Pero, sobre todo, necesita aprender a reconocer y valorizar los procesos de cambio mínimo o de mejoría ligera, dado que, por las características de estas familias, éstos pueden llegar a ser imperceptibles. (Vega, 1997, p. 169)

\section{Contextos Profesionales de Cambio}

Existen diversos contextos profesionales de cambio en la intervención con FMP. En la Tabla 1 se han adaptado los contenidos propuestos por Lamas (1997) desde la revisión bibliográfica y reflexión realizada por los autores de este artículo.

Los diversos contextos de intervención (de evaluación, asistencial, de consulta, informativo, terapéutico y de control) que aquí se muestran, iluminan el hallazgo empírico de que los profesionales no siempre se relacionan con la FMP en un mismo ámbito de interacciones o momento del proceso. 
A cada contexto se le asocia un campo de significados posibles, que encuadran los intercambios profesionales (y no profesionales) entre los diversos involucrados en el proceso de intervención. Asimismo, cada contexto tiene objetivos, modalidades, metodologías y técnicas que le son propias.

Esto no es idéntico a decir que sólo se actúa en un nivel, o que un servicio debe acotarse a un único contexto de intervención. Es evidente que en la práctica coexisten en paralelo o incluso entrelazadamente diversos campos de práctica profesional (por ejemplo, asistencial y terapéutico son dos contextos relativamente frecuentes).

Más bien se trata de que el profesional cuente con un esquema conceptual clarificador de las características, posibilidades y limitaciones de cada contexto, y así pueda esforzarse por evitar confundir los problemas que enfrenta, los recursos que necesita, y las competencias que requiere para poder intervenir adecuadamente en cada caso.

\section{Instrumentos de Trabajo}

Vega (1997) ha identificado como instrumentos de trabajo con FMP: (1) el profesional como persona; (2) el equipo profesional; (3) las otras redes de intervención; y (4) la formación y la supervisión. Para maximizar el aporte de cada uno de estos instrumentos, debe realizarse un trabajo cíclico de reflexión, entrenamiento y evaluación del estado actual y deseado, según el contexto particular de la intervención. El autoconocimiento y una supervisión constante que explore las vivencias emocionales del operador, permiten prevenir el desgaste profesional asociado al trabajo con FMP. Al respecto, cabe señalar que

Este trabajo supone un impacto emocional intenso, con el consiguiente riesgo para el profesional, dado que se debe enfrentar a las descripciones particulares de la vida que tienen estas familias: sus historias de supervivencia, sus desastres reiterativos, sus urgencias, sus miserias, sus estilos alternativos de vida familiar y sus soluciones, que suelen ofender las creencias, valores y costumbres del profesional. La necesidad del autoconocimiento personal de éste supone aceptar la existencia de una involucración emocional, con sus consiguientes riesgos en todo proceso de ayuda. (Vega, 1997, p. 171)

\section{Estrategias de Intervención}

Para abordar la complejidad de la FMP, existen estrategias inespecíficas transversales, como el uso de la empatía (Cunningham \& Henggeler, 1999) $\mathrm{y}$ una actitud de genuino interés y apoyo «que venga del corazón», y no sólo como resultado de una obligación laboral, manteniendo la esperanza en que el cambio es posible, a pesar de las crisis y dificultades que surjan en el proceso. Esta actitud ha sido mencionada como una variable crucial en el éxito con FMP, según la percepción de los propios usuarios de servicios sociales (Knel-Paz \& Ribner, 2000). Además, existen estrategias específicas, como las que se detallan en la Tabla 2.

\section{Tabla 2}

\section{Estrategias especificas para trabajar con FMP}

$\Rightarrow$ Aumentar estructura de las intervenciones

$\Rightarrow$ Implementar contratos de participación

$\Rightarrow$ Aprovechar motivación al cambio en las crisis

$\Rightarrow$ Desarrollar metas de tratamiento realistas

$\Rightarrow$ Evitar un enfoque de "apaga-incendios"

$\Rightarrow$ Derivar cuidadosamente a otros servicios

$\Rightarrow$ Ayudar a la familia a valorar la intervención

Uno de los desafíos centrales del trabajo con FMP es lograr su adherencia e involucramiento en los espacios propuestos. Las FMP no suelen llevar una agenda o recordar citaciones con profesionales; simplemente, hay otras necesidades que llaman con mayor urgencia su atención y energías. Para Cunningham y Henggeler (1999), la intervención no puede progresar sin involucramiento, siendo indicadores de su presencia: (1) altas tasas de asistencia; (2) cumplimiento de "tareas para la casa"; (3) activación emocional durante las sesiones; y (4) progreso hacia las metas acordadas. Como indicadores de problemas de involucramiento se mencionan: (1) constantes dificultades y obstáculos para fijar citaciones; (2) inasistencias frecuentes; (3) no seguir los planes de intervención; y (4) mentiras sobre datos importantes (Cunningham \& Henggeler, 1999).

Para enfrentar este problema, McNeil y Herschell (1998) proponen intensificar la estructura de las citaciones, definiendo mecanismos para recordar fechas y horarios y para trasladarse hasta el centro, llamando por teléfono el día anterior o entregando invitaciones personalmente o a través del correo. $\mathrm{Al}$ intervenir con FMP se sugiere definir un contrato escrito de asistencia y participación en el programa, estipulando las características del tratamiento, duración, objetivos, qué resultados puede esperar el (los) 
cliente(s) y fechas relevantes (McNeil \& Herschell, 1998). Esto ayuda a mejorar la comprensión del cliente sobre el proceso, clarificando expectativas mutuas y minimizando conflictos de planificación e implementación.

Otra estrategia sugerida, es aprovechar la elevada motivación al cambio que tienen estas familias en los momentos de crisis, debido al sentido de urgencia con que los vivencian. Sin embargo, su desorganización característica puede hacer cambiar las prioridades rápidamente, por lo que los equipos deben estar preparados para usar el impulso como palanca a favor del proceso de cambio, en la dirección definida como "foco" de la intervención.

Como no es posible abordar todos los problemas de una FMP al mismo tiempo, McNeil y Herschell (1998) proponen escoger una o dos áreas con el mayor grado de especificidad posible e intervenir mediante la negociación de metas trabajables con la familia o miembros de la familia que estén en tratamiento. Este enfoque, que trabaja pequeñas experiencias de éxito desde una mirada a los recursos y capacidades resilientes, ayuda a transformar un locus de control externo en uno interno, con el consecuente empoderamiento de las familias y sus miembros (Juby \& Rycraft, 2004).

Relacionado con la estrategia de escoger un número pequeño de metas realistas, está el evitar la trampa asociada a las múltiples crisis, dinámica que puede llevar al profesional o equipo a confundirse fácilmente y a perder el hilo conductor de su intervención, diluyéndose con ello sus efectos potencialmente beneficiosos. McNeil y Herschell (1998) sugieren acoger empáticamente las quejas o la información relacionada con la nueva crisis, pero limitar el tiempo dedicado a escucharlas. En vez de dejarse llevar por cada nuevo episodio de derrumbe individual o familiar, el profesional que está interviniendo debe recordarse a sí mismo el foco que ha definido para el abordaje de esta familia y volver oportunamente a él.

Es relevante reconocer que ningún profesional ni equipo puede lidiar por sí solo con todos los pedazos de solución que se necesitan para superar estas dinámicas autoperpetuantes (Haz, Díaz \& Raglianti, 2002). En consecuencia, los otros pedazos de solución deben necesariamente buscarse en la Red, suscitando entonces el problema de la sobreintervención profesional antes descrito. Para evitar esto, McNeil y Herschell (1998) proponen priorizar las derivaciones, tal como se priorizan las metas de la intervención; el trabajo con estas familias se beneficia de una estrategia secuencial de derivación, más que simultánea, porque la simultaneidad satura sus recursos de respuesta y colabora al fracaso de la intervención.

Por otra parte, es necesario trabajar en estas familias la valorización de la terapia y los espacios de intervención como una prioridad (McNeil \& Herschell, 1998). Frente a necesidades de alimentación, apuros económicos y otras, es difícil considerar prioritario asistir y permanecer en terapia o en las intervenciones propuestas. Es por ello que el profesional debe generar una experiencia positiva en sus clientes, usando el elogio honesto como principal herramienta; también se ha denominado a esta estrategia "dar regalos" terapéuticos, como trasmitir esperanza y fe en el cambio o normalización de problemas y sentimientos (Cunningham \& Henggeler, 1999). Para los padres y madres, hijos e hijas de estas familias, estos espacios pueden ser los únicos momentos en que experimentan la sensación de sentirse bien acerca de algún aspecto de sí mismos. Por ello, el elogio honesto puede reparar su autoestima y ser un piso de vinculación desde el cual fortalecer la adherencia al tratamiento.

Asimismo, el mostrar periódicamente los avances obtenidos, facilita la sensación subjetiva de avance, lo que a su vez redunda en una mayor adherencia al tratamiento (McNeil \& Herschell, 1998). Esto puede realizarse, por ejemplo, mediante "reuniones de celebración de logros", o a través de la entrega de "diplomas de reconocimiento" de habilidades parentales o de logros en la superación de ciertos síntomas (White \& Epston, 1990 citado en Lamas, 1997).

Finalmente, se sugiere desarrollar las intervenciones (si el contexto lo permite) en una atmósfera libre de juicios y condenas, en donde se estimule la expresión libre de las vivencias y emociones. El respeto y la empatía se han mencionado como actitudes terapéuticas inespecíficas de alto impacto sobre estas familias (Cunningham \& Henggeler, 1999). Esta pauta, en una dinámica plagada de problemas, introduce un elemento novedoso y constructivo, aliviando la vivencia de estrés y facilitando una actitud de apertura al cambio. En la práctica esto se puede realizar expresando a los padres que ellos están haciendo lo mejor que pueden en una situación realmente difícil (pues lo es por definición); lo que viven sería un desafío para cualquier familia, por lo que el trabajo profesional se redefine desde ayudarlos a desarrollar habilidades y competencias para enfrentar con éxito dichos desafíos (McNeil \& Herschell, 1998). 
Tabla 3

Intervención con Familias Multiproblemáticas

\begin{tabular}{lll}
\hline Característica & Foco propuesto a intervenir & Resultado Esperado \\
\hline $\begin{array}{l}\text { Polisintomatología } \\
\text { (múltiples problemas, crisis recurrentes } \\
\text { y situación crónica de adversidad) }\end{array}$ & Resiliencia personal y familiar & $\begin{array}{l}\text { Desplegar recursos y fortalezas personales } \\
\text { y familiares (por sobre un énfasis en la } \\
\text { patología) }\end{array}$ \\
$\begin{array}{l}\text { Desorganización } \\
\text { (en estructura, dinámica y }\end{array}$ & Organización familiar & Lograr una mejor organización familiar \\
comunicación) & & \\
$\begin{array}{l}\text { Abandono de las funciones parentales } \\
\text { Aislamiento }\end{array}$ & Apego y vinculación & $\begin{array}{l}\text { Fortalecer los vínculos padre/madre } \\
\text { - hijos(as) }\end{array}$ \\
Pobreza & Red & $\begin{array}{l}\text { Mayor integración a la red formal e } \\
\text { informal }\end{array}$ \\
& Necesidades básicas & $\begin{array}{l}\text { Construcción de una plataforma mínima } \\
\text { de recursos y servicios }\end{array}$ \\
\hline
\end{tabular}

\section{Propuesta}

A lo largo de este artículo, se comprendió a la familia multiproblemática como un sistema vulnerable de alto riesgo para todos sus miembros. En su conjunto, el resquebrajamiento de la matriz de cariño y protección, que generan el entramado de vulneración en que se despliegan las funciones parentales y dinámicas de interacción, puede facilitar la emergencia de los múltiples problemas que afectan a estas familias. Estos problemas se concatenan en un patrón de transmisión transgeneracional altamente tóxico y costoso para el niño o niña, su familia y la sociedad de la que forman parte.

De lo antedicho se desprende la importancia fundamental de diseñar, implementar, evaluar y difundir programas que sean efectivos en interrumpir la disfuncionalidad actual y la transmisión transgeneracional de este patrón en las FMP.

La efectividad de las intervenciones con FMP ha sido escasa y errática en diversos lugares del mundo. EE.UU. es el país que ha desarrollado la mayor cantidad de investigación de calidad y a gran escala para medir el impacto real de las intervenciones sociales en estas familias, documentando el bajo efecto que suelen tener los programas sociales al enfrentar la enorme complejidad de esta tarea con los mecanismos tradicionales de intervención psicosocial (Lindsey, Martin \& Doh, 2002).

Las intervenciones profesionales con FMP, tanto en el ámbito social como clínico, se han desprendido de un enfoque centrado en el déficit: para cada problema, se plantea una solución especializada y desarticulada de otros recursos, con un método de trabajo compensatorio. Este enfoque ha demostrado ser de escasa efectividad, al concentrarse en aquello de lo cual la familia debe alejarse, más que focalizarse en aquello hacia lo cual la familia debe dirigirse (Sousa, Ribeiro \& Rodrigues, 2006).

Como resultado de la investigación bibliográfica realizada, la reflexión de los autores y la participación de uno de ellos en el desarrollo del Marco Conceptual Orientador de la Sociedad Protectora de la Infancia, en el presente artículo se propone una concepción del trabajo con familias multiproblemáticas que asocia a cada característica definida, un foco a intervenir, anclado en las posibilidades de cambio más que en los déficit y patologías.

Como se observa en la Tabla 3, se propone que el profesional y los equipos contrapongan al despliegue caleidoscópico de problemáticas, una mayor atención a los recursos y potencialidades no actualizadas ${ }^{2}$. El concepto unificador de las acciones profesionales en esta línea es el de resiliencia, que se entiende como "la capacidad de sobreponerse a la adversidad de forma creativa, transformando el suceso negativo, y potencialmente dañino, en un aprendizaje enriquecedor" (Protectora de la Infancia, 2007, p. 29).

Es importante recalcar que un acercamiento a la polisintomatología de la FMP desde sus recursos y

2 Este énfasis es concordante con la propuesta de Sousa, Ribeiro y Rodrigues (2006) de integrar a la práctica el enfoque centrado en los problemas con el enfoque centrado en las fortalezas como orientaciones complementarias en el trabajo con FMP. 
fortalezas, no implica negar la presencia de severos problemas de salud mental o minimizar la gravedad de situaciones de violencia intrafamiliar, entre muchas otras problemáticas. El método de trabajo con FMP propuesto reconoce la presencia de dichas situaciones, derivando a instancias especializadas cuando sea necesario y trabajando coordinadamente con otros equipos, pero al mismo tiempo pone de relieve la tarea fundamental de identificar y potenciar activamente áreas libres de patología, daño y disfuncionalidad. Desde dicho reconocimiento, la FMP puede construir capacidad de resiliencia para enfrentar, por transferencia de aprendizaje, otras problemáticas similares e incluso, dependiendo de su plasticidad, problemáticas de índole diversa. En la intervención con FMP ambas caras de la moneda deben hacerse presentes: (1) el problema; (2) los recursos; y (3) un entrenamiento en cómo usar los recursos para afrontar los problemas.

Asimismo, frente a la desorganización imperante, el profesional busca intervenir la estructura y dinámica de las familias, con la finalidad de mejorar su funcionamiento global. El concepto guía en este caso es el de organización familiar, que se comprende como "el resultante dinámico del entrecruzamiento entre aspectos estructurales, relacionales e históricos de un sistema familiar, que se despliegan en la vida cotidiana y se ponen a prueba en situaciones de estrés" (Protectora de la Infancia, 2007). Las estrategias propuestas por McNeil y Herschell (1998) se muestran como intervenciones fructíferas para abordar esta variable.

En tercer lugar, el abandono de las funciones parentales, si bien forma parte del trabajo sobre la organización familiar, se entiende como fuertemente ligado a una intervención sobre los vínculos que unen a padres y madres (o figuras significativas) con sus hijos e hijas, por lo que el apego emerge como concepto gravitante. El apego entonces, se entenderá como "un vínculo afectivo reductor de estrés, específico y estable, que une al bebé (o niño/a) con una persona significativa para él, en la búsqueda de la protección y cuidados, confianza y estimulación cariñosa, que necesita para su desarrollo" (Protectora de la Infancia, 2007, p. 29). En la actualidad existen intervenciones diseñadas específicamente para potenciar el desarrollo de un apego seguro o mejorar patrones de apego inseguros con prometedoras aplicaciones a esta población (Juffer, BakermansKranenburg \& van IJzendoorn, 2005).

En cuarto lugar, al aislamiento (y/o dependencia de los servicios sociales) que caracteriza a estas familias, oponemos un trabajo dirigido a su integración a la red formal e informal. Cabe señalar que se entiende por red al "entramado de vínculos interpersonales e institucionales, reales y potenciales, que permiten y facilitan la integración comunitaria del niño y su familia, revirtiendo condiciones de aislamiento social, y cumpliendo funciones de apoyo emocional, consejo, regulación y adaptación social, ayuda material y de servicios, y acceso a nuevos contactos" (Protectora de la Infancia, 2007, p. 29). Consideramos no sólo el trabajo directo con la familia, sino también los indispensables espacios de coordinaciones que deben existir entre todos los actores involucrados en el proceso de ayuda, según se señaló en este artículo, si han de evitarse los efectos iatrogénicos de la intervención profesional.

Finalmente, la pobreza pone en evidencia las múltiples necesidades que enfrentan estas familias en su vida cotidiana. Las necesidades básicas se entienden en este trabajo como "la plataforma mínima de recursos y servicios, necesaria para el desarrollo biopsicosocial de un niño y para el adecuado ejercicio de las funciones parentales" (Protectora de la Infancia, 2007) y se visualizan como un soporte para todos los otros elementos señalados. Avanzar en este aspecto requiere el concurso de todos los actores comunitarios, privados, políticos y gubernamentales implicados; en este plano, la organización de mesas territoriales puede contribuir notoriamente cuando se desarrollan con respeto, voluntad y responsabilidad social.

El trabajo con FMP demanda una visión ecosistémica de la intervención; potenciar las funciones parentales no puede concebirse aisladamente de una mirada al apego, por una parte, y a la organización familiar (en sus vertientes de estructura, dinámica y competencias parentales), por otra. Fortalecer el apego seguro no puede hacerse sin observar el componente de resiliencia relacional que ahí se pone en juego. Asimismo, la superación de la pobreza en una FMP no resulta viable sin una metodología que incluya la coordinación con los servicios y recursos de la red.

Es importante destacar que la disfuncionalidad de la FMP no es una característica intrínseca a ésta de forma aislada, sino que se constituye en el resultado de la interacción patogénica de diversos sistemas, incluidos los sistemas profesionales de ayuda, que no logran coordinarse adecuadamente en su actuar. El desmembramiento y el efecto de atomización y desvinculación que suele observarse en estas familias es el resultado de una historia fragmentada, tanto desde los severos trastornos de apego con que se da 
inicio a la fundación de la familia, como desde el involucramiento parcializado de los múltiples agentes de intervención ( $\mathrm{y}$ así sucesivamente).

Es por ello que el profesional que intervenga con familias multiproblemáticas debe realizar un esfuerzo por organizar su acercamiento al caso desde las características presentadas, sin olvidar que las variables que se entrecruzan en la gestación de estos problemas "están fragmentadas por todo el espacio humano e institucional, de manera tal que las soluciones deberán ser, consecuentemente, planteamientos que convoquen los trozos de solución que invariablemente estarán dispersos en los más insospechados nodos de la red" (Haz, et al., 2002 , p. 27). Para que este esfuerzo sea efectivo, debe cumplir básicamente con tres requisitos (que sintetizan lo expuesto en este artículo):

1. Co-construir un foco de intervención y conservarlo como punto de referencia a medida que la familia va presentando su abanico de dificultades. Esto evita en parte la dependencia crónica al acotar el trabajo profesional a ciertos objetivos, dejando otros fuera y además otorga organización a una familia que se caracteriza por no tenerla;

2. Aprender a leer los pequeños avances y entrenarse en resaltarlos con entusiasmo, para sí mismo y para la familia, estructurando un proceso que marque claramente los hitos de logro de la familia (lo que además permite retornar a estos puntos de éxito cuando se instalan las recaídas y retrocesos); y

3. Coordinar activamente el trabajo con los otros profesionales y servicios de la red que estén involucrados o que vayan a involucrarse en un futuro cercano (por ejemplo, mediante un trabajo de derivaciones vinculares).

Además de lo anteriormente señalado, y desde nuestra experiencia con familias multiproblemáticas en la Sociedad Protectora de la Infancia, es importante enfatizar la necesidad de incorporar espacios para recordar periódicamente el encuadre fijado en los contratos iniciales. Asimismo, estos espacios sirven para entregar retroalimentación respecto al grado de avance, estancamiento y/o retroceso en los diversos acuerdos negociados al comienzo del proceso.

El método de trabajo aquí propuesto debe ser tomado como una orientación más que como un modelo, por cuanto no cuenta con respaldo empírico que sustente su efectividad. Por otra parte, cabe destacar que sus conceptos están anclados en enfoques teóricos y tradiciones de investigación con amplio reconoci- miento y consenso en la comunidad científica, por lo que cabría esperar que futuras investigaciones y experiencias de aplicación práctica, entreguen respaldo a las ideas desarrolladas en este artículo.

Se hace necesario estimular la investigación en países de habla hispana sobre las características específicas que adopta la familia multiproblemática en estos contextos, sobre metodologías de intervención desarrolladas y su efectividad, y sobre las percepciones de las familias y los profesionales en relación a estas variables.

Es urgente desarrollar un cuerpo de conocimientos fundamentado en la investigación científica rigurosa de estos factores para retroalimentar a los encargados de las políticas públicas, de la administración de las organizaciones sociales y a los equipos profesionales que intervienen directamente con familias multiproblemáticas en riesgo social.

Derivado de este conocimiento, construido a partir de la realidad sociocultural específica de los países latinoamericanos, sería posible mejorar el diseño de los programas, la capacitación de los equipos, la coordinación de las redes y en definitiva, la intervención profesional con familias multiproblemáticas y en riesgo social.

\section{Referencias}

Aldaz-Carroll, E. \& Moran, R. (2001). Escaping the poverty trap in Latin America: The role of family factors. Cuadernos de Economía, 38(114), 155-190.

Bauman, Z. (2001). La posmodernidad y sus descontentos. Madrid: Akal.

Barudy, J. (1998). El dolor invisible de la infancia: Una lectura ecosistémica del maltrato infantil. Barcelona: Paidós.

Barudy, J. \& Dantagnan, M. (2005). Los buenos tratos a la infancia. Parentalidad, apego y resiliencia. Barcelona: Gedisa.

Cancrini, L., De Gregorio, F. \& Nocerino, S. (1997). Las familias multiproblemáticas. En M. Coletti \& J. Linares, La intervención sistémica en los servicios sociales ante la familia multiproblemática: La experiencia de Ciutat Vella (pp. 45-82). Barcelona: Paidós Terapia Familiar.

Colapinto, J. (1995). Dilution of family process in social services: Implications for treatment of neglectful families. Family Process, 34(2), 59-74.

Cortina, A. (1998). Hasta un pueblo de demonios. Ética pública y sociedad. Madrid: Taurus.

Cunningham, P. \& Henggeler, S. (1999). Engaging multiproblem families in treatment: Lessons learned troughout the development of multisystemic therapy. Family Process, 38 (3) $265-281$.

Cyrulnik, B. (2002). Los patitos feos. La resiliencia: Una infancia infeliz no determina la vida. Barcelona: Gedisa.

De Paúl, J., Múgica, P. \& Alday, N. (1997). Evaluación del programa de apoyo a familias en situaciones de desprotección infantil en Castilla y León. España: Junta de Castilla y León.

Ditzel, L. \& Maldonado, M. (2004, octubre). El apoyo a la familia en sus funciones primordiales, un eje fundamental de la 
reforma del Sename. Ponencia presentada en el XIX Congreso Panamericano del Niño, Santiago, Chile.

Ehrenberg, A. (1999). La fatiga de ser uno mismo. Depresión y sociedad. Buenos Aires: Nueva Visión.

Finzi, R., Ram, A., Har-Even, D., Shnit, D. \& Weizman, A. (2001). Attachment styles and aggression in physically abused and neglected children. Journal of Youth and Adolescence, 30(6), 769-786.

Glaser, D. (2002). Emotional abuse and neglect (psychological maltreatment): A conceptual framework. Child Abuse and Neglect, 26, 697-714.

Gracia, E. \& Musitu, G. (1993). El maltrato infantil: Un análisis ecológico de los factores de riesgo. Madrid: Ministerio de Asuntos Sociales.

Gracia, E. \& Musitu, G. (2003). Social isolation from communities and child maltreatment: A cross-cultural comparison. Child Abuse and Neglect, 27, 153-168.

Haz, A.M., Díaz, D. \& Raglianti, M. (2002). Un desafío vigente para el Psicólogo Clínico: El entendimiento de estrategias de intervención para abordar problemas de riesgo. Revista Terapia Psicológica, 20(1), 23-28.

Haz, A.M. \& Matus, T. (2006). Desafios asociados a la profesionalización de los servicios de las organizaciones de acción social: El caso de los programas que trabajan con la infancia gravemente vulnerada en sus derechos. Proyecto Fondecyt. Documento no publicado.

Hughes, D. (2004). An attachment-based treatment of maltreated children and young people. Attachment \& Human Development, 6(3), 263-278.

Hurst, N., Sawatzky, D. \& Pare, D. (1996). Families with multiple problems through a Bowenian lens. Child Welfare, 75(6), 693-708.

Juby, C. \& Rycraft, J. (2004). Family preservation strategies for families in poverty. Families in Society, 85(4), 581-588.

Juffer, F., Bakermans-Kranenburg, M. \& van IJzendoorn, M. (2005). Enchancing children's socio-emotional development: A review of intervention studies. En D.M. Teti (Ed.), Handbook of research methods in developmental science (pp. 213-232). Oxford: Blackwell.

Knel-Paz, C. \& Ribner, D. (2000). A narrative perspective on "doing" for multiproblem families. Families in Society: The Journal of Contemporary Human Services, 81(5), 475-482.

Lamas, C. (1997). Los primeros contactos. En M. Coletti \& J. Linares (Ed.), La intervención sistémica en los servicios sociales ante la familia multiproblemática: La experiencia de Ciutat Vella (pp. 83-124). Barcelona: Paidós Terapia Familiar.

Linares, J. (1997). Modelo sistémico y familia multiproblemática. En M. Coletti \& J. Linares (Ed.), La intervención sistémica en los servicios sociales ante la familia multiproblemática: la experiencia de Ciutat Vella (pp. 23-44). Barcelona: Paidós Terapia Familiar.

Lindsey, D., Martin, S. \& Doh, J. (2002). The failure of intensive casework services to reduce foster care placements: An examination of family preservation studies. Children and Youth Services Review, 24(9-10), 743-775.

Minuchin, P., Colapinto, J. \& Minuchin, S. (2000). Pobreza, institución, familia. Buenos Aires: Amorrortu.

Matos, A. \& Sousa, L. (2004). How multiproblem families try to find support in social services. Journal of Social Work Practice, 18 (1), 65-80.

McNeil, C. \& Herschell, A. (1998). An essay for practitioners. Treating multi-problem, high stress families: Suggested strategies for practitioners. Family Relations, 47(3), 259-262.

Moncher, F. (1995). Social isolation and Chile-abuse risk. Families in society, 76(7), 421-433.

Navarro, M. (2002). La intervención psicosocial con familias multiproblemáticas: La perspectiva ecológica. Tesis Doctoral, Universidad de Valencia, España.

Protectora de la Infancia (2007). Memoria Anual 2006. Santiago: Sociedad de Asistencia y Capacitación.

Roditti, M. (2005). Understanding communities of neglectful parents: Child caregiving networks and child neglect. Child Welfare, 84 (2), 277-298.

Rozas, G. (1999). Familia y pobreza dura. Revista de Psicología de la Universidad de Chile, 3 (1), 83-94.

Sharlin, S. \& Shamai, M. (1995). Intervention with families in extreme distress (FED). Marriage \& Family Review, 21 (1-2), 91-122.

Sluzki, C. (1996). La red social: Frontera de la práctica sistémica. Barcelona: Gedisa.

Sousa, L. (2005). Building on personal networks when intervening with multiproblem poor families. Journal of Social Work Practice, 19 (2), 163-179.

Sousa, L. \& Eusébio, C. (2005). When multi-problem poor individuals' values meet pratitioners' values! Journal of Community \& Applied Social Psychology, 15, 353-367.

Sousa, L., Ribeiro, C. \& Rodrigues, S. (2006). Intervention with multi-problem poor clients: Towards a strengths-focused perspective. Journal of Social Work Practice, 20 (2), 189-204.

Vega, S. (1997). Instrumentos de trabajo. En M. Coletti \& J. Linares (Eds.), La intervención sistémica en los servicios sociales ante la familia multiproblemática: La experiencia de Ciutat Vella (pp. 167-200). Barcelona: Paidós Terapia Familiar.

Walsh, F. (2004). Resiliencia familiar: Estrategias para su fortalecimiento. Buenos Aires: Amorrortu.

Woodward, L. \& Fergusson, D. (2002). Parent, child and contextual predictors of childhood physical punishment. Infant and Child Development, 11, 213-235. 